\title{
Research on the Relationship between Organizational Justice and Organizational Citizenship Behaviors
}

\author{
Peiying $\mathrm{Wu}^{1, \mathrm{a}^{*}}$ and Lifen Chen ${ }^{2, \mathrm{~b}}$ \\ ${ }^{12}$ School of Economics and Management, Nanjing University of Science and Technology, \\ Nanjing 210094, China \\ aywupeiying@126.com, bjustclf@163.com \\ *The corresponding author
}

\begin{abstract}
Organizational citizenship behaviors have been widely referred in the field of management, and there are many researches about OCB, among which, one of the most significant issue is the relationship between organizational justice and OCB. Organizational justice can affect individual's role cognition and behavior, and the occurrence of individual's OCB is based on the background of organizational justice. This paper has reviewed the researches on the relationship between organizational justice and $\mathrm{OCB}$ at home and abroad, and according to the different emphasis, aim to give some helpful suggestions on indigenous research.
\end{abstract}

Keywords: Organizational justice; Organizational citizenship behaviors; Relationship

\section{组织公平与组织公民行为的关系研究}

\section{吴佩莹 ${ }^{1}$ 陈丽芬 ${ }^{2}$}

（南京理工大学 经济管理学院、企业管理系，江苏南京 210094）

摘要: 组织公民行为已经在管理领域的研究中被广泛的提及, 关于组织公民行为的研究有很多, 其中一项重要的就是组 织公平与组织公民行为的关系研究, 组织公平影响着个体的角色认知和行为, 员工的组织公民行为产生, 是作为公平意义上 的一个自发性选择。本文主要总结了国内外关于组织公平和组织公民行为的关系研究, 通过整理出关于这一研究的文献综述, 更好的梳理这方面研究的脉络, 以及对未来的本土化研究有一个大致方向性的理解。

关键词: 组织公平; 组织公民行为; 关系研究

中图分类号: F241 文献标识码: A

引言

在过去的 40 年里, 组织公平是组织行为研究领域最受关注的, 其被定义为 “员工在组织中感知到的 公平, 如他们是否公平地从他们的付出中交换得到奖励; 他们是否在程序上和人际上获得了公平的待遇。” ${ }^{[1]}$ 员工在结果、程序以及交换过程中对公平的感知很大程度上影响了他们对组织支持的态度和行为。Spector 等人在关于组织公平影响的元分析中, 揭示了组织公平, 如分配公平、程序公平、互动公平, 对员工的工 作满意度、组织承诺、工作绩效、对组织的信任均有着正向关系。 ${ }^{[2]}$ Colquitt 基于社会交换理论, 也总结 了组织公平通过社会交换质量（信任、组织承诺、组织支持感、 LMX) 对员工的任务绩效、周边绩效有着 显著的影响。[3]

在众多组织公平的结果变量中, 组织公民行为可以算得上是一个比较热门的研究主题。组织公民行为 是一种自愿的员工行为, 这是超出了正常的职责或者规定之外的行为。 ${ }^{[4]}$ 很多之前的研究者在很大程度上 在社会交换理论关系下解释组织公民行为, 也就是说, 如果员工有与其组织较高质量的交换关系, 这种交 换关系是以相互信任、互利互惠为特征的。与组织公民行为的基本原理一致, 组织公平在交换关系情境下, 
在员工和组织之间起着作用, 因为员工的公平感知会受他们与组织的交换是否公平的影响。同样的, 鉴于 社会交换理论是解释组织公平和组织公民行为的一个重要的也是共同的基础, 因此，关于组织公平和组织 公民行为的研究也就繁荣起来。

\section{1 组织公平}

组织公平的研究开始于 1965 年亚当斯对于分配公平的研究, 亚当斯在《社会交换中的不公平》一文 中的提出了公平理论, 后来的学者们在这启示下, 对组织公正也进行了大量的研究, 开始从他们的角度, 正式定义组织公平。比较有代表性的研究者有 Colquitt、Greenber 等人。从学者们的研究总结来看, 组织 中的公平分为两个层面, 第一个层面是客观状态的组织公平, 在这个层面上, 人们可以通过对各种组织制 度的不断改善和发展, 建立相应的制度和程序, 采取适当的手段和措施, 来实现组织公平。第二个层面是 组织公平感, 也就是组织中的成员对组织公平的主观感受，是一种态度。 ${ }^{[4]}$

从内容上区分, 目前认可比较广泛的观点是组织公平可以分成分配公平、程序公平和互动公平。分配 公平主要表现在员工付出和薪酬回报之间的关系, 指一个人对于自己的收入或分派的公平知觉。程序公平 是强调在资源分配过程中使用的程序及过程公平, 没有弄虚作假。互动公平倾向于双向沟通, 指组织程序 中个人的人际传播, 人际互动的敏感程度。

对比中西方的研究, 在组织公平这一构念上, 中西方学者还是有各自侧重的方面。西方更关注个人与 组织之间工具性关系, 这种关系基于公平交换原则, 是对物质资源的公平分配, 并最终指向资源分配结果 的公平。而国内学者对基于不同文化、制度和组织构成之上的组织公平感的结构维度及其对组织结果变量 的影响也进行了相关研究。[5,6,7]

\section{2 组织公民行为}

1938 年，Barnard 提出，组织是合作力量的联合体，员工的合作意愿对正式结构是一个关键因素，否 则正式结构就成了空壳。Organ 认为, Barnard 的 “合作的意愿” 与 Roethlisberger 和 Dicksion（1964）对 霍桑实验中 “非正式组织” 的研究包含了组织公民行为思想的精髓, 这可说是组织公民行为研究的溯源。

Smith, Organ, Near（1983）及 Organ（1988）正式提出组织公民行为的概念，认为组织公民行为即 员工的自主行为, 在整体上有利于组织功效的发挥。组织公民行为体现出员工的自主性, 这种行为在角色 和工作描述中没有强制要求, 雇用合同中也没有包括, 是个人意志的选择。

后来, Organ 在以往研究的基础上, 将组织公民行为划分为五个维度: 利他主义, 即乐于帮助同事, 预防和解决与工作相关问题的行为; 运动员精神, 即员工在非理想化的情境下, 不抱怨, 仍然为了组织的 利益坚持做事; 尽责行为, 即严肃认真、尽心尽力完成工作; 公民道德, 即员工作为组织中的一个公民应 有的道德行为; 文明礼貌, 即在工作中尊重他人, 礼让他人。当然也有很多学者给出了不同的维度分类标 准, 如 Podsakoff 的六维论, 国内学者樊景立的十一维度论等等。 ${ }^{[8]}$

\section{3 组织公平和组织公民行为关系研究}

\section{1 国外研究}

关于组织公平和组织公民行为的关系, 国外学者起步较早, 研究较多, 而且研究的点比较细致。早在 上个世纪 70 年代, Dittrch 和 Carroll(1979)就发现工作公平与薪酬公平的知觉与角色行为显著相关, 这也是 
这两者关系研究的雉形初现。Organ(1988)认为, 员工对组织公平的认知和组织公民行为之间存在正相关关 系。进入 90 年代, 关于这一关系的研究开始迅速蓬勃起来。

Niehoff \& Moorman (1993)认为: 当组织在分配资源和决定奖惩措施, 员工个人主观认知感到不公平, 为平衡这种不公平状态而决定减少个人投入时，一般不会选择减少与工作绩效有直接关系、可能影响到他 未来获得酬劳的投入，而选择减少不会影响到个人酬劳、代价相对较低的组织公民行为方式 ${ }^{[9]}$, 这一研究 结论更完整的将组织公平、组织公民行为和工作绩效三者之间的关系呈现了出来。而且 Moorman 等人一 直在这方面做着持续的研究, 其在后续的研究中发现程序公平与组织公民行为的相关性大于分配公平与组 织公民行为之间的相关性。Konnovsky 和 Pugh(1994)通过社会交换理论预测组织公民行为, 认为程序公平 能够直接影响到上级主管对员工信任的建立, 进而影响组织公民行为的产生。SkarliCki 和 Latham(1996)以 加拿大工会为研究对象, 证实员工的组织公平感与组织公民行为之间的正相关关系。 ${ }^{[10]}$ Dewi S. 通过实证 研究, 验证了组织公平通过组织承诺, 对员工的组织公民行为产生正向影响。[11]类似的, 很多研究也都直 接或间接的验证了组织公平与组织公民行为之间的关系。[12,13,14]

近十多年来, 在原有的基础上, 关于组织公平和组织公民行为的研究更加具体。Naumann 和 Bennett(2000)沿着 Moorman 的研究脉络, 将组织公平和组织公民行为的具体内容进行结合研究, 发现程序 公平与助人行为显著相关。Levy 和 Haworth(2001)研究表明组织公民行为是员工了解组织绩效评估系统、 相信组织中的程序是公平的体现。William, Pitre 和 Zainuba(2001)分别从分配公平、程序公平、互动公平 三种层面测量其与组织公民行为之间的关系, 结果显示均显著, 但是互动公平的显著性更高。 $\mathrm{LiX}, \mathrm{Qu} \mathrm{Y}$, Ren H 的一篇关于酒店行业员工组织公民行为的实证研究指出, 分配公平对员工 OCB 没有显著影响, 而程 序公平对公民道德有着显著的正向关系，互动公平对运动员精神、团结同事有显著的正向关系。[15]

\section{2 国内研究}

我国在这方面的研究算是比较晚, 而且研究的比较零散, 没有形成一定的体系, 但是也出现了一些有 价值的成果。刘亚、龙立荣(2002)的研究指出, 领导公平对组织承诺、组织公民行为有显著影响, 程序公 平对组织承诺有显著影响, 但是对组织公民行为没有显著影响, 领导解释对组织公民行为存在显著影响, 分配公平对组织承诺存在显著影响。[16]

王长福(2005) 从员工绩效评估公平的构念出发, 对员工绩效评估公平感、组织承诺与组织公民行为的 关系进行了研究, 组织承诺作为部分中介。张鹏(2007)的研究的也验证了组织信任、组织承诺在组织公平 与组织公民行为之间的中介作用。但是, 陈景刚(2005) 以国有企业员工为研究对象, 分析了组织公平和组 织公民行为的关系, 并考虑了组织信任的中介作用。组织公平与 OCB 各个子维度之间的关系显著, 但是 信任的中介作用不是很明显。

也有学者探讨了组织支持感中介作用下的组织公平与 OCB 之间的关系, 组织支持的不同维度, 其中 介作用也不一样。 ${ }^{[17]}$ 苗仁涛, 孙健敏, 刘军 (2012) 同时探讨了组织支持和组织公平对员工组织公民行为 之间的关系, 以工作态度为中介。 ${ }^{[18]}$ 近几年来, 学者们开始更具体探讨 OCB 的发展困境, 并着重研究其 提升机制。[19,20]

从国内学者们目前的研究来看, 主要是分成两大类别, 一类是将组织公平或者组织公民行为分成若干 子构念, 探讨子构念之间的直接影响关系。第二类就是以组织信任、组织承诺、工作满意度等作为中介变 
量, 探讨它们在组织公平和组织公民行为之间的中介作用, 分析组织公平与组织公民行为之间的间接关系。

\section{4 讨论}

通过对相关文献的回顾和梳理可以看出, 国内外学者对组织公平、组织公民行为的研究成果是非常丰 富的。从组织公平、组织公民行为概念的提出, 到形成各自的研究体系, 再到二者关系研究的蓬勃发展, 是学者们不断探索、不断假设验证的过程。

现有关于组织公平与组织公民行为之间关系的研究，在理论框架的设计上，大部分是仅仅着眼于组织 公平与组织公民行为之间的间接关系, 对组织公平与组织公民行为之间关系的中间机制因素及其相应的内 在机理的关注度相对不够。立足于公平理论和社会交换理论的基本观点, 进行组织公平与组织公民行为之 间关系的直接作用机制的探讨，应该作为公平理论和社会交换理论未来的努力方向。

最后, 在中国这样一个重视人情关系, 具有高权力距离的国家, 互动公平、领导公平对员工行为的影 响也具有重要的研究价值。因此, 本土化的组织公平与组织公民行为关系研究也是未来研究的重点。

\section{参考文献:}

[1] Greenberg J. Organizational Justice: Yesterday, Today, and Tomorrow[J]. Journal of Management: Official Journal of the Southern Management Association, 1990, 16(2):399-432.

[2] Cohen-Charash Y, Spector P E. The role of justice in organizations: A meta-analysis: Erratum[J]. Organizational Behavior \& Human Decision Processes, 2002, 89:1215-1215.

[3] Colquitt J A, Scott B A, Rodell J B, et al. Justice at the millennium, a decade later: a meta-analytic test of social exchange and affect-based perspectives.[J]. Journal of Applied Psychology, 2013, 98(2):199-236.

[4] 郑景丽, 郭心毅. 组织公平对员工组织公民行为影响的实证研究 [J]. 北京理工大学学报(社会科学版), 2016, $18(1): 82-88$.

[5] 赵旻. 绩效评估公平感的影响因素研究[D]. 南京理工大学, 2014.

[6] 王重鸣, 徐小军. 我国公务员绩效评估公平性结构的实证研究[J]. 心理科学, 2007, 30(2):277-280.

[7] 陈丽芬, 江卫东. 绩效评估公正感问题研究:结构、前因与效应[M]. 科学出版社, 2014.

[8] 张敏. 不同文化背景下企业员工组织公民行为维度的对比研究[J]. 商业经济, 2010(5):88-89.

[9] Moorman R H, Niehoff B P. Does Perceived Organizational Support Mediate the Relationship between Procedural Justice and Organizational Citizenship Behavior?[J]. Academy of Management Journal, 1998, 41(3):351-357.

[10] Skarlicki D P, Latham G P. Increasing citizenship behavior within a union: A test of organizational justice theory[J]. Journal of Applied Psychology, 1996, 81(2):161-169.

[11] Dewi S. Analysis Effect of Organizational Justice on Organizational Commitment and Organizational Citizenship Behavior (OCB) at PDAM Tirta Pakuan in Bogor[J]. MT - Economic and Management, 2012.

[12] Perreira T, Berta W. Increasing OCB: the influence of commitment, organizational support and justice[J]. Strategic HR Review, 2015, 14(1/2):13-21.

[13]Zeinabadi H, Salehi K. Role of procedural justice, trust, job satisfaction, and organizational commitment in Organizational Citizenship Behavior (OCB) of teachers: Proposing a modified social exchange model[J]. Procedia - Social and Behavioral Sciences, 2011, 29(1):1472-1481.

[14] Gotlib T. Multifoci Organizational Justice, Organizational Citizenship Behavior, and Counterproductive Work Behavior: The Mediating Effects of Emotions[J]. Dissertations \& Theses - Gradworks, 2011.

[15] Li X, Qu Y, Ren H. Examining the impact of organizational justice and servant leadership on OCB[C]// Service Systems and Service Management (ICSSSM), 2012 9th International Conference on. IEEE, 2012:23-27.

[16] 李晔, 龙立荣, 刘亚.组织公平感研究进展[J].心理科学进展,2003(11):78-84.

[17] 曹慧, 梁慧平. 员工公平感对组织公民行为的影响：组织支持的中介作用 $[J]$. 科技管理研究, 2010 , 
$30(14): 186-189$.

[18] 苗仁涛, 孙健敏, 刘军. 基于工作态度的组织支持感与组织公平对组织公民行为的影响研究 $[\mathrm{J}]$. 商业经济与管 理, 2012, $251(9): 29-40$.

[19]杨斌，陈坤. 组织公民行为概念的发展困境及其突破线索探讨 $[J]$. 外国经济与管理，2012 (3) :31-39.

[20] 翁清雄, 刘勇, 张增田, 等. 基层公务员组织公民行为提升机制研究——基于社会心理动因视角 [J]. 软科学, $2014(11): 86-89$.

\section{References:}

[1] Greenberg J. Organizational Justice: Yesterday, Today, and Tomorrow [J]. Journal of Management: Official Journal of the Southern Management Association, 1990, 16(2):399-432.

[2] Cohen-Charash Y, Spector P E. The role of justice in organizations: A meta-analysis: Erratum [J]. Organizational Behavior \& Human Decision Processes, 2002, 89:1215-1215.

[3] Colquitt J A, Scott B A, Rodell J B, et al. Justice at the millennium, a decade later: a meta-analytic test of social exchange and affect-based perspectives.[J]. Journal of Applied Psychology, 2013, 98(2):199-236.

[4] Zheng Jingli, Guo Xinyi. An Empirical Study on the Effect of Organizational Justice on Employee's Organizational Citizenship Behavior [J]. Journal of Beijing Institute of Technology (Social Science Edition), 2016, 18 (1): 82-88.

[5] Zhao Min A Study on the Influencing Factors of Fairness in Performance Evaluation [D]. Nanjing University of Science and Technology, 2014.

[6] Wang Chongming, Xu Xiaoqing An Empirical Study on the Fairness Structure of Performance Evaluation of Civil Servants in China [J]. Psychological Science, 2007, 30 (2): 277-280.

[7] Chen Lifen, Jiang Weidong A Study on the Problem of Fairness of Performance Evaluation: Structure, Antecedency and Effect [M]. Science Press, 2014.

[8] Zhang Min A Comparative Study on Organizational Citizenship Dimension of Employees in Different Cultural Backgrounds [J]. Commercial Economics, 2010 (5): 88-89.

[9] Moorman R H, Niehoff B P. Does Perceived Organizational Support Mediate the Relationship between Procedural Justice and Organizational Citizenship Behavior? [J]. Academy of Management Journal, 1998, 41(3):351-357.

[10] Skarlicki D P, Latham G P. Increasing citizenship behavior within a union: A test of organizational justice theory [J]. Journal of Applied Psychology, 1996, 81(2):161-169.

[11]Dewi S. Analysis Effect of Organizational Justice on Organizational Commitment and Organizational Citizenship Behavior (OCB) at PDAM Tirta Pakuan in Bogor [J]. MT Economic and Management, 2012.

[12] Perreira T, Berta W. Increasing OCB: the influence of commitment, organizational support and justice [J]. Strategic HR Review, 2015, 14(1/2):13-21.

[13]Zeinabadi H, Salehi K. Role of procedural justice, trust, job satisfaction, and organizational commitment in Organizational Citizenship Behavior (OCB) of teachers: Proposing a modified social exchange model [J]. Procedia - Social and Behavioral Sciences, 2011, 29(1):1472-1481.

[14]Gotlib T. Multifoci Organizational Justice, Organizational Citizenship Behavior, and Counterproductive Work Behavior: The Mediating Effects of Emotions [J]. Dissertations \& Theses - Gradworks, 2011. 
[15] Li X, Qu Y, Ren H. Examining the impact of organizational justice and servant leadership on OCB $[\mathrm{C}] / /$ Service Systems and Service Management (ICSSSM), 2012 9th International Conference on. IEEE, 2012:23-27.

[16] Liye, Long Lirong,Liuya. Advances in Organizational Justice [J]. Advances in Psychological Science, 2003 (11): 78-84.

[17] Caohui, Liang Huiping the Impact of Employee Fairness on Organizational Citizenship: The Mediating Role of Organizational Support [J]. Science and Technology Management Research, 2010, 30 (14): 186-189.

[18] Miao Rentao, Sun Jianmin, Liujun Research on the Influence of Organizational Support and Organizational Justice on Organizational Citizenship Behavior Based on Job Attitude [J]. Commercial Economics and Management, 2012, 251 (9): 29-40.

[19] Yangbin, Chenkun on the Development Dilemma of Organizational Citizenship Concept and Its Breakthrough [J]. Foreign Economy and Management, 2012 (3): 31-39.

[20] Wen Qingxiong, Liuyong, Zhang Zengtian,etc. A Study on the Mechanism of Citizens 'Behavior Enhancement in Grassroots Civil Servants' Organizations - From the Perspective of Social Psychological Motivation [J]. Soft Science, 2014 (11): 86-89. 